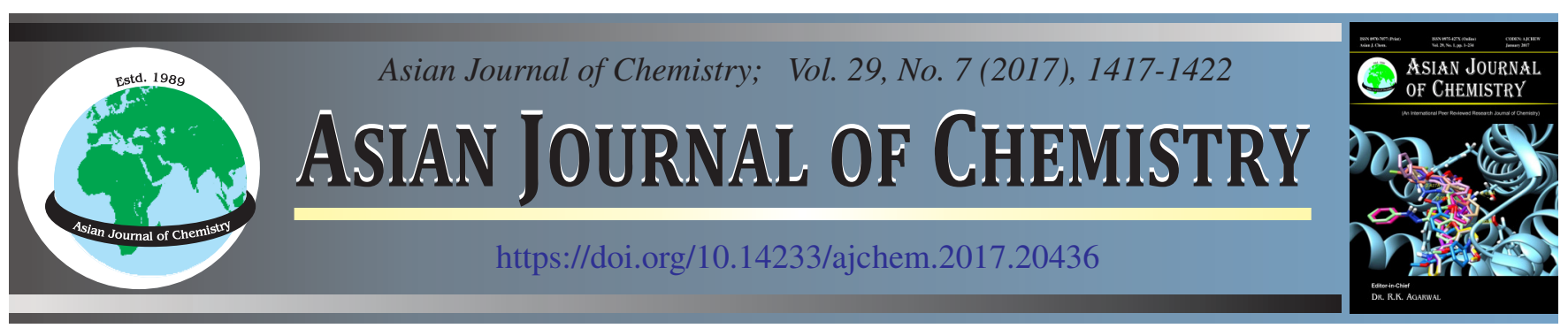

\title{
Synthesis of Silver Nanoparticles by Reduction of Silver Ion with $\boldsymbol{m}$-Hydroxybenzoic Acid
}

\author{
Gusrizal Gusrizal $^{1,2}$, SRI Juari Santosa ${ }^{1, *}$, Eko Sri Kunarti ${ }^{1}$ and Bambang Rusdiarso ${ }^{1}$
}

${ }^{1}$ Department of Chemistry, Faculty of Mathematics and Natural Sciences, Universitas Gadjah Mada, Sekip Utara, PO Box BLS 21, Yogyakarta 55281, Indonesia

${ }^{2}$ Department of Chemistry, Faculty of Mathematics and Natural Sciences, Universitas Tanjungpura, Jl. Prof. Dr. H. Hadari Nawawi, Pontianak 78124, Indonesia

*Corresponding author: E-mail: sjuari@ugm.ac.id

\begin{abstract}
In this study, we demonstrated the use of $m$-hydroxybenzoic acid in the synthesis of stable silver nanoparticles (AgNPs). Besides working as a reducing agent, $m$-hydroxybenzoic acid also simultaneously played a role as a capping agent. In the synthesis of AgNPs, silver nitrate was first mixed with the $\mathrm{pH}$-adjusted $m$-hydroxybenzoic acid solution. The mixture was then heated in a boiling water bath. The formation of AgNPs was indicated by the appearance of yellow colour usually completed in $15 \mathrm{~min}$. The reducing ability of $m$-hydroxybenzoic acid was affected by its $\mathrm{pH}$ and concentration. At a mole ratio of silver nitrate and $m$-hydroxybenzoic acid 1:10, the reaction produced AgNPs with the average size of $19 \pm 9 \mathrm{~nm}$. Stability test over a period of 18 weeks showed that AgNPs were highly stable with the intensity of surface plasmon resonance peak only reduced $2.0 \%$. This experiment indicates the potency of AgNPs.
\end{abstract}

Keywords: Silver nanoparticles, Synthesis, $m$-Hydroxybenzoic acid.

\section{INTRODUCTION}

Nanomaterial, a material with any external dimension or having the internal structure in the size range of 1-100 $\mathrm{nm}$, has been used in many products. Application of nanomaterial follows its unique properties including high surface to volume ratio, high surface energy and unique mechanical, thermal, electrical, magnetic and optical behaviours. These properties are different compared to their bulk material with the same chemical composition $[1,2]$. Over the past few years, the published papers show that there has been a significant increase of research to explore nanoparticles. Nanoparticles may occur in different shapes such as a sphere, cube, tetrahedron, octahedron, bar, spheroid, bipyramid, decahedron, wire, rod, plate, disc and hollow structures. There is a correlation between the properties of nanoparticles with their shape and size [3].

Among the metal nanoparticles, silver nanoparticles (AgNPs) have taken great of interest. Silver nanoparticles have been known as an antibacterial agent and have been used in the biomedical application, water treatment, food production, textile industries and consumer goods. Experimental data show that the antibacterial properties of AgNPs depend on the size and shape of nanoparticles [4,5]. The size and shape of AgNPs can be controlled by the synthesis method [6].
A large number of methods are now available for the synthesis of AgNPs. In chemical reduction method, the precursor of silver, reducing agent and stabilizer or capping agent should be available in the synthesis of AgNPs [3]. Sodium borohydride and sodium citrate are commonly used in the reduction of silver ion to generate AgNPs. Polymers such as polyvinyl alcohol stabilize the AgNPs during and after formation of nanoparticles [7]. Development of synthesis method to produce AgNPs with different reducing and capping agent has been reported [8-11].

The objective of this study is to produce stable AgNPs using $m$-hydroxybenzoic acid as reducing agent without addition of any capping agent. Previous work showed that $o$ - and $p$ hydroxybenzoic acids could be used in the formation of AgNPs [12]. In this paper, we describe the method in producing of stable AgNPs using $m$-hydroxybenzoic acid as reducing and simultaneously capping agents which is much more convenient than the previous work using $o$ - and $p$-hydroxybenzoic acids.

\section{EXPERIMENTAL}

Silver nitrate (Merck) $m$-hydroxybenzoic acid (SigmaAldrich) and sodium hydroxide (Merck) were used as received and no further purification was performed. UV-visible spectrophotometer (Shimadzu UV-PharmaSpec), Fourier transmission 
infrared spectrophotometer (Shimadzu FTIR Prestige-21) and transmission electron microscope (JEOL JEM-1400) were used in the characterization of the resulted AgNPs. The sample for FTIR measurement was obtained by centrifugation of AgNPs colloid at $13.000 \mathrm{rpm}$ by IEC MB Centrifuge.

Synthesis of silver nanoparticles: Synthesis of AgNPs was performed similarly to previous work [12]. Briefly, $5 \mathrm{~mL}$ of silver nitrate solution was added to $5 \mathrm{~mL}$ of $\mathrm{pH}$-adjusted $m$ hydroxybenzoic acid in a test tube. The mixture of silver nitrate and $m$-hydroxybenzoic acid was heated in boiling water bath and then cooled in tap water. Resulted AgNPs were stored at room temperature and normal laboratory condition for monitoring their stability. Reducing ability of $m$-hydroxybenzoic acid was studied by reacting various concentrations of silver nitrate $\left(0.5\right.$ to $\left.2.5 \times 10^{-4} \mathrm{M}\right)$ with a constant concentration of $m$-hydroxybenzoic acid at $1.0 \times 10^{-3}$ and $1.0 \times 10^{-2} \mathrm{M}$. This synthesis of AgNPs was performed at two different $\mathrm{pHs}$ of the $m$-hydroxybenzoic acid solution (pH 11 and 12). Sodium hydroxide solution was used to adjust the $\mathrm{pH}$ of $m$-hydroxybenzoic acid solution.

Characterization of silver nanoparticles: The formation of AgNPs was monitored by UV-visible spectrophotometer using $1 \mathrm{~cm}$ optical path length quartz cuvette and 200-800 nm wavelength range. The FTIR spectra were obtained by scanning the $\mathrm{KBr}$ plate of AgNPs in the range of $4000-400 \mathrm{~cm}^{-1}$. The solid AgNPs sample for FTIR measurement was prepared by drying at $65{ }^{\circ} \mathrm{C}$ the centrifugation-recovered AgNPs from their colloidal solution. Preparation of sample for TEM analysis was performed by immersing the copper grid into AgNPs colloid and then drying at room temperature. The accelerating voltage of $120 \mathrm{kV}$ was used in taking the image. The scale provided in the micrograph was used in the calculation of the particle size.

\section{RESULTS AND DISCUSSION}

Reaction time for the formation of silver nanoparticles: Determination of time required for the formation of AgNPs was performed by addition of $1 \times 10^{-4} \mathrm{M}$ silver nitrate to $1 \times$ $10^{-3} \mathrm{M} m$-hydroxybenzoic acid solution at $\mathrm{pH} 11$. The mixture of silver nitrate and $m$-hydroxybenzoic acid was then heated in boiling water bath and the UV-visible spectra were collected at different interval time reaction. Silver nanoparticles formation was confirmed by the appearance of yellow colour in the mixture of $m$-hydroxybenzoic acid and silver nitrate. The observed intense yellow colour was due to the surface plasmon resonance, which could be measured by UV-visible spectrophotometer [13]. The intensity of colour and peak of the spectra related to the concentration of resulted AgNPs.

All reaction showed the formation of AgNPs. Although the intensity of peaks was different with time reaction, all peaks were centered at 410-420 $\mathrm{nm}$. The maximum result of the AgNPs formation, as shown in Fig. 1, was achieved at 15 min of reaction time. There was different in the rate of AgNPs formation using different isomers of hydroxybenzoic acid if compared with previous data [12]. Formation of AgNPs using $o$ - and $p$-hydroxybenzoic acids took longer time (2 and $1 \mathrm{~h}$ ) than $m$-hydroxybenzoic. The order of consuming time for the reaction followed the order $o->p$ - $>m$-hydroxybenzoic acid.

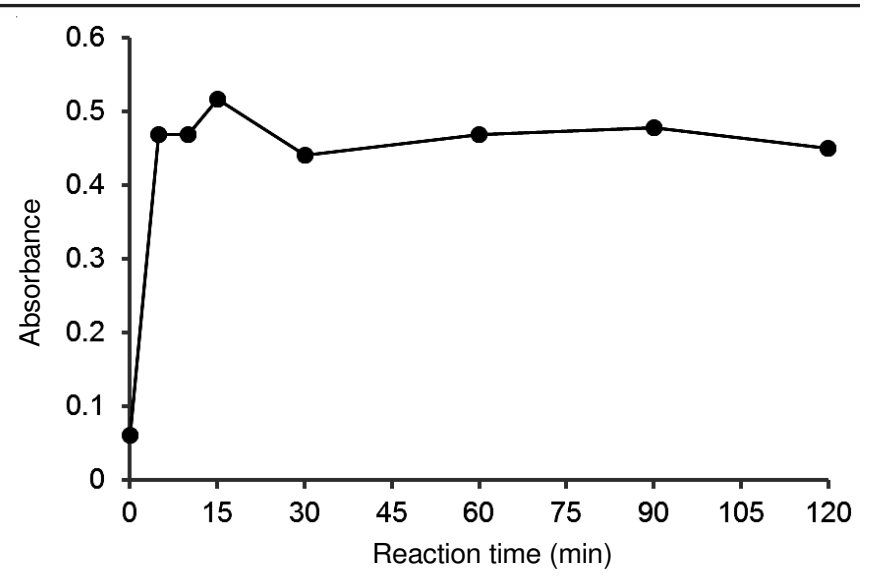

Fig. 1. Plot of the peak intensity of AgNPs versus time during the synthesis

Reducing ability of $\boldsymbol{m}$-hydroxybenzoic acid: The reducing ability of $m$-hydroxybenzoic acid was determined by plotting the intensity of surface plasmon resonance peak versus the initial concentration of silver nitrate used in the reaction. This relationship is described by a linear line model with the equation of $y=a x+b$, where $y$ is the intensity of maximum peak, $x$ is initial concentration of silver nitrate, $b$ is a constant and $\mathrm{a}$ is the gradient of the line representing the reducing ability of $m$-hydroxybenzoic acid. To evaluate the reducing ability of $m$-hydroxybenzoic acid, we used two concentration of $m$-hydroxybenzoic acid $\left(1.0 \times 10^{-3}\right.$ and $1.0 \times$ $10^{-2} \mathrm{M}$ ) and two $\mathrm{pH}$ of the $m$-hydroxybenzoic acid solution (pH 11 and 12). The initial concentration of silver nitrate was varied from 0.5 to $2.5 \times 10^{-4} \mathrm{M}$. For all reactions, the spectra centered at around $400-430 \mathrm{~nm}$ and the intensity of spectra increased with the increasing the concentration of silver nitrate. Surface plasmon spectra of all reaction are shown in Fig. 2.

By plotting the peak intensity versus initial concentration of silver nitrate provided in Fig. 2, we obtained several equations. The equation of each reaction conditions was $\mathrm{y}=7218 \mathrm{x}$ $-0.2\left(R^{2}=0.99\right), y=7099 x-0.2\left(R^{2}=0.99\right), y=5490 x-0.2$ $\left(R^{2}=0.96\right)$ and $y=3802 x\left(R^{2}=0.99\right)$ for data in Fig. 2A, 2B, $2 \mathrm{C}$ and $2 \mathrm{D}$ respectively. The gradient of the linear line represents the reducing ability of $m$-hydroxybenzoic acid. The maximum reducing ability of $m$-hydroxybenzoic acid could be achieved when the reaction was performed using $1.0 \times 10^{-3} \mathrm{M} m$-hydroxybenzoic acid with its $\mathrm{pH}$ was adjusted to 11 . The increase of $\mathrm{pH}$ and concentration of $m$-hydroxybenzoic acid decreased the reducing ability of $m$-hydroxybenzoic acid.

The resonance of hydroxybenzoic acid isomers was believed influencing their reducing ability. The resonance for $o$ - and $p$-hydroxybenzoic acids is occurred to the exo-ring system, in contrast for $m$-hydroxybenzoic acid, where the resonance takes place only to the endo-ring system. In the case of $o$-hydroxybenzoic acid, internal hydrogen bonding may also have a contribution. Hence, the $m$-hydroxybenzoic acid had the highest reducing ability. By comparing with previous data [12], the order of hydroxybenzoic isomers reducing ability followed $m>p>o$-hydroxybenzoic acid. This order agrees with the previous data published by Simic et al. [14]. The reducing ability also correlated with the rate of reaction for the formation of AgNPs using these reducing agents. 

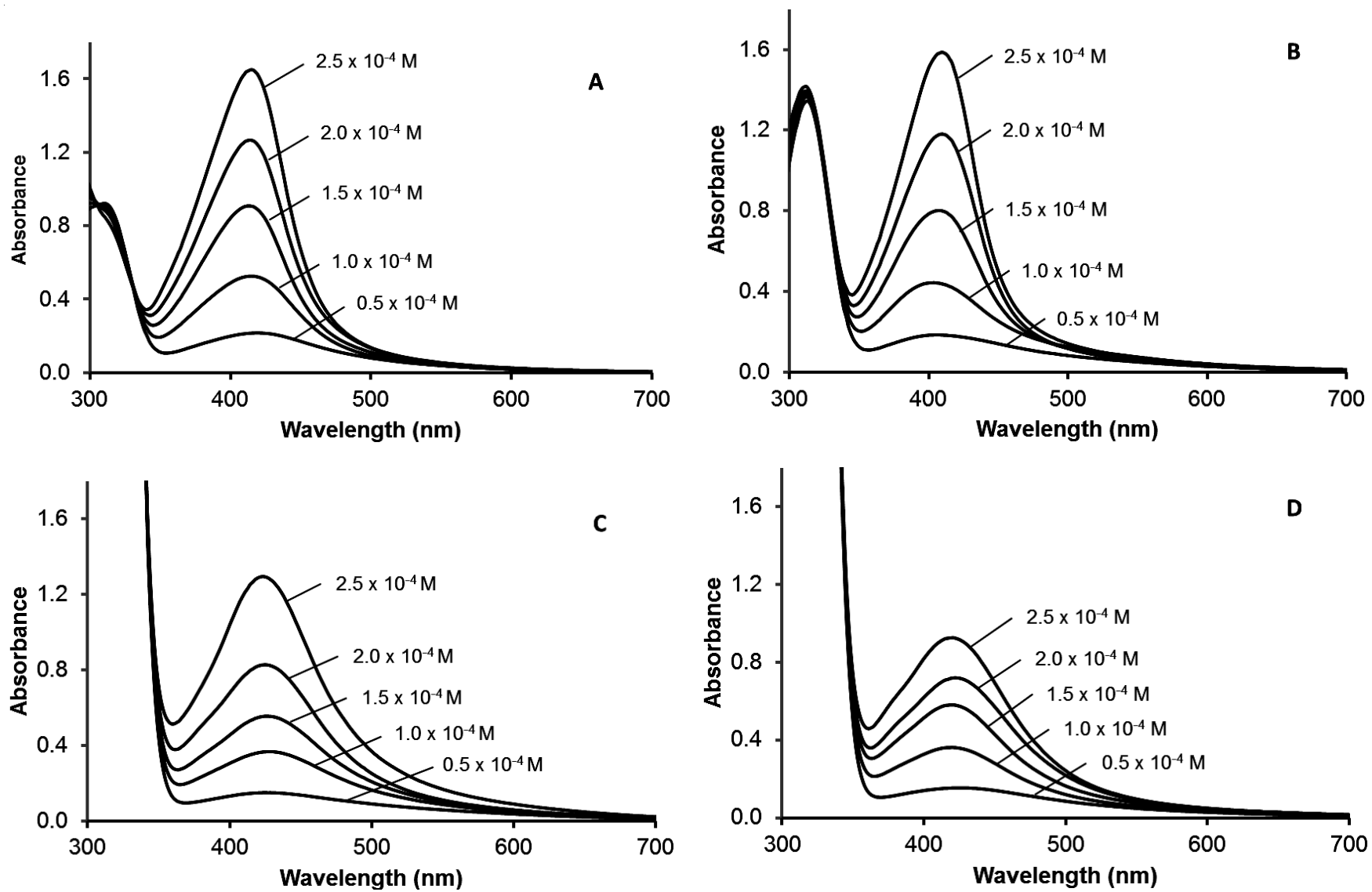

Fig. 2. Surface plasmon resonance spectra of AgNPs synthesized using different initial concentration of silver nitrate (A) [ $m$-hydroxybenzoic acid $]=1.0 \times 10^{-3} \mathrm{M}, \mathrm{pH}=11 ;(\mathrm{B})[\mathrm{m}$-hydroxybenzoic acid $]=1.0 \times 10^{-3} \mathrm{M}, \mathrm{pH}=12 ;(\mathrm{C})[\mathrm{m}$-hydroxybenzoic acid $]=1.0 \times 10^{-2} \mathrm{M}, \mathrm{pH}$ $=11 ;(\mathrm{D})[\mathrm{m}$-hydroxybenzoic acid $]=1.0 \times 10^{-2} \mathrm{M}, \mathrm{pH}=12$

Stability of silver nanoparticles: The stability of AgNPs was studied at the duration of 18 weeks storage at a normal laboratory condition. Silver nanoparticles used for stability test were synthesized using $1.5 \times 10^{-4} \mathrm{M}$ silver nitrate and two concentrations of $\mathrm{pH}$ 11-adjusted $\mathrm{m}$-hydroxybenzoic acid (1.0 $\times 10^{-3}$ and $\left.1.0 \times 10^{-2} \mathrm{M}\right)$. Observation after 18 weeks showed that the colour of AgNPs was visually still identic with that of fresh synthesized AgNPs. Fig. 3 shows the profile of AgNPs surface plasmon resonance spectra. Compared to the fresh synthesized AgNPs, there was only $2 \%$ of peak intensity reduction but with no observable shift of $\lambda_{\max }$ from $416 \mathrm{~nm}$. The similar result was also found for the synthesized AgNPs using $1 \times 10^{-2} \mathrm{M} m$-hydroxybenzoic acid. No observable shift of $\lambda_{\max }$ at $424 \mathrm{~nm}$ and only a $1.5 \%$ reduction in the peak intensity were found. These data suggested that the resulted AgNPs were highly stable.

Since we did not use additional capping agent to stabilize the resulted AgNPs, it is proposed that $m$-hydroxybenzoic acid simultaneously serves as both reducing and capping agent. Silver nanoparticles were capped by $m$-hydroxybenzoic acid
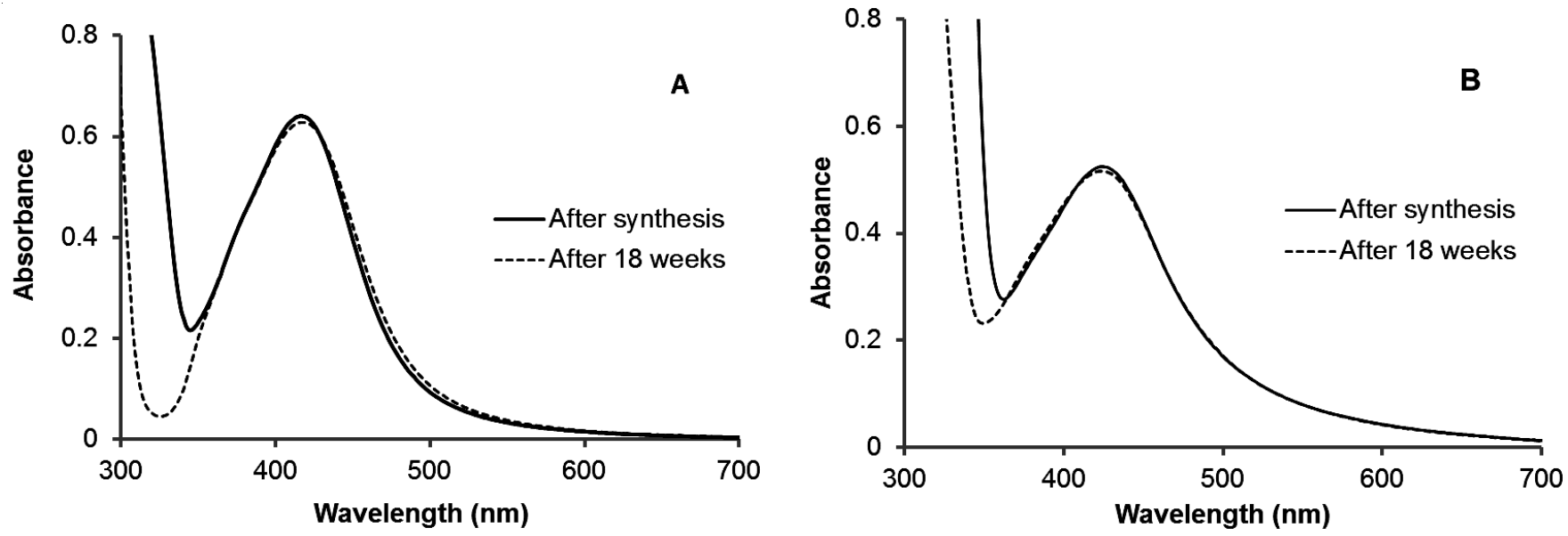

Fig. 3. Surface plasmon resonance spectra of AgNPs after a period of 18 weeks; (A) [ $m$-hydroxybenzoic acid] $=1.0 \times 10^{-3} \mathrm{M}$; $(\mathrm{B})$ $[m$-hydroxybenzoic acid $]=1.0 \times 10^{-2} \mathrm{M}$ 
and resulted in the stabilization effect. The attachment of $m$ hydroxybenzoic acid on the surface of AgNPs was predicted through its carboxyl functional group. The FTIR spectra of $m$-hydroxybenzoic acid (spectra not shown here) showed that the intensity of the peak at around $1700 \mathrm{~cm}^{-1}(\mathrm{C}=\mathrm{O}$ vibration) was higher than the peak at around $1600 \mathrm{~cm}^{-1}(\mathrm{C}=\mathrm{C}$ aromatic vibration). It was different with the spectra of $m$-hydroxybenzoic acid capped AgNPs (Fig. 4). The spectra showed no observable peak at around $1700 \mathrm{~cm}^{-1}$. No observable peak around $1700 \mathrm{~cm}^{-1}$ indicated the contribution of the carboxyl group in the complexation with AgNPs [15]. Previously published data also support that hydroxybenzoic acid attach to the AgNPs through its carboxyl group and providing stabilization effect [16,17].

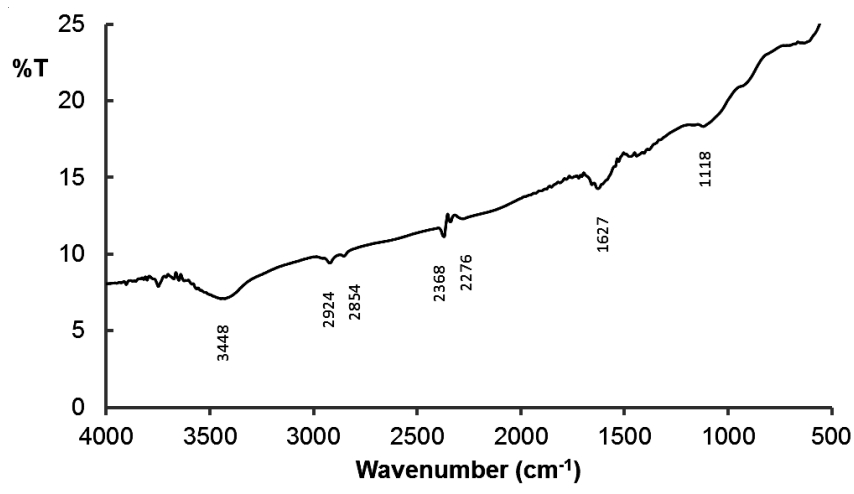

Fig. 4. FTIR spectra of $m$-hydroxybenzoic acid capped AgNPs [ $m$ hydroxybenzoic acid $]=1.0 \times 10^{-3} \mathrm{M},\left[\mathrm{AgNO}_{3}\right]=2.0 \times 10^{-4} \mathrm{M}, \mathrm{pH}$ $=11$

Effect of the concentration of $m$-hydroxybenzoic acid: The stability studies show that $m$-hydroxybenzoic acid does not only play a role in reducing but also in capping the resulted AgNPs. To determine the effective mole ratio of silver nitrate and $m$-hydroxybenzoic acid, the synthesis was performed using a different concentration of $m$-hydroxybenzoic acid with a constant initial concentration of silver nitrate $\left(2.0 \times 10^{-4} \mathrm{M}\right)$. The concentration of $m$-hydroxybenzoic acid was varied from $0.5 \times 10^{-4}$ to $1.0 \times 10^{-2} \mathrm{M}$. Fig. 5 shows the intensity of the peak of AgNPs produced with different concentration of $m$-hydroxybenzoic acid. It can be seen that the maximum result was exhibited by the reaction using $2 \times 10^{-3} \mathrm{M}$ of $m$-hydroxybenzoic acid where the mole ratio of silver nitrate and $\mathrm{m}$ hydroxybenzoic acid was 1:10. The amount of formed AgNPs tended to decrease with the increasing of the concentration of $m$-hydroxybenzoic acid over the mole ratio of $1: 10$.

To understand the effect of an excess of $m$-hydroxybenzoic acid concentration on the particle size, we performed the synthesis based on the result presented in Fig. 5. The $2.0 \times$ $10^{-4} \mathrm{M}$ silver nitrate and two concentration of $\mathrm{pH} 11$-adjusted $m$-hydroxybenzoic acid $\left(2 \times 10^{-3}\right.$ and $\left.1 \times 10^{-2} \mathrm{M}\right)$ were used. In these reactions, the mole ratio of silver nitrate and $\mathrm{m}$ hydroxybenzoic acid were 1:10 and 1:50. The images and particles size distributions are shown in Fig. 6. Reduction using mole ratio 1:10 produced the particles size ranged from 8 to $62 \mathrm{~nm}$ with the average $19 \pm 9 \mathrm{~nm}$. On the other hand, particles size ranged from 13 to $87 \mathrm{~nm}$ with the average $31 \pm$ 15 produced by the reaction using mole ratio 1:50. The result

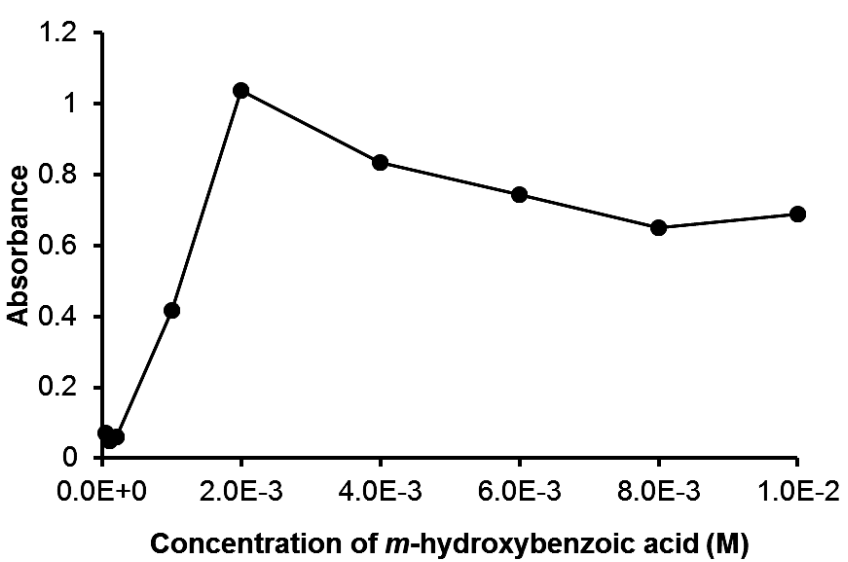

Fig. 5. Plot of AgNPs peak intensity and concentration of $m$-hydroxybenzoic acid used in the synthesis of AgNPs

showed that the particle size and polydispersity of AgNPs increased by the increasing concentration of $m$-hydroxybenzoic acid.

The results of TEM analysis support the measurement using UV-visible spectrophotometer (Fig. 7). The red shift of $\lambda_{\max }$ from 415 to $425 \mathrm{~nm}$ and an increase of the spectra at wavelength around $600 \mathrm{~nm}$ indicated that AgNPs produced using a higher concentration of $m$-hydroxybenzoic acid had a larger particle size and high polydispersity. The correlation between particle size and polydispersity with the intensity of spectra at longer wavelength are also supported by other published data $[5,18]$.

The mole ratio of silver nitrate and $m$-hydroxybenzoic acid influenced the size of AgNPs. An excess of $m$-hydroxybenzoic acid produced larger particles size with higher polydispersity. The ratio of silver nitrate and capping agent plays an important role in determining the shape and size of AgNPs [19]. Increasing the concentration of reducing agent that also simultaneously serves as capping agent has a tendency to produce larger particles [20].

In this paper, we also suggest that $m$-hydroxybenzoic and $p$-hydroxybenzoic acid have the same way in action as a capping agent. As proposed in our previous paper for $p$-hydroxybenzoic [12], $m$-hydroxybenzoic acid creates an arrangement of their molecules providing the space for the growth of AgNPs. Higher concentration of $m$-hydroxybenzoic acid results in the larger arrangement of the molecules so providing a larger space for particles growth. It is why an excess of $m$-hydroxybenzoic acid results in larger particles.

\section{Conclusion}

We developed a rapid method for synthesizing of stable AgNPs using $m$-hydroxybenzoic acid without additional capping agent. The reducing ability of $m$-hydroxybenzoic acid was influenced by its $\mathrm{pH}$ and concentration. The effective mole ratio of silver nitrate and $m$-hydroxybenzoic acid was 1:10 at the $\mathrm{pH}$ of reaction equals to 11 . The average size of resulted AgNPs synthesized with these reaction parameters was $19 \pm 9$ $\mathrm{nm}$. Increasing the concentration of $m$-hydroxybenzoic acid enhanced the polydispersity and the size of particles $(31 \pm 15$ $\mathrm{nm})$. The AgNPs were stable over the period of 18 weeks at normal laboratory condition. 

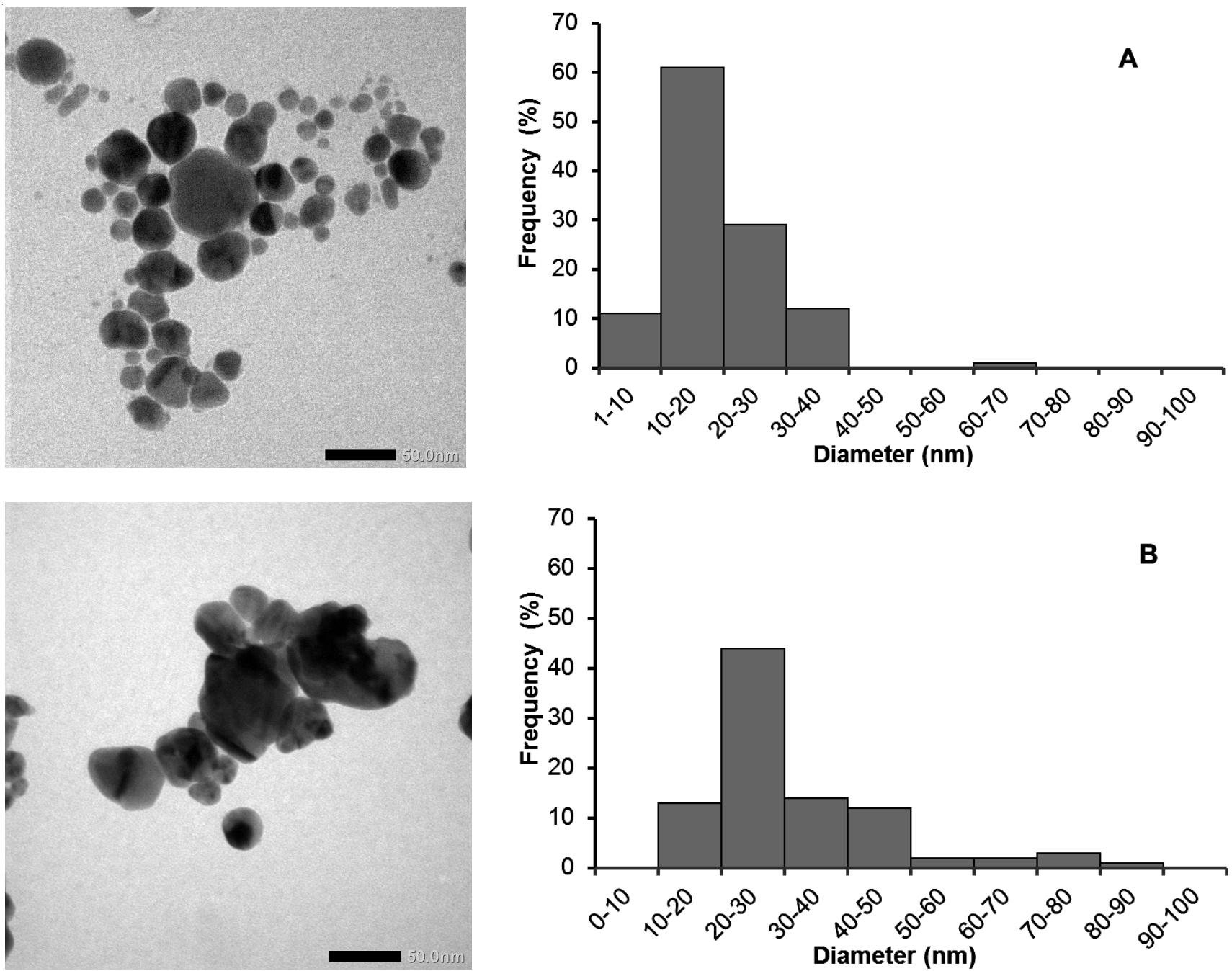

Fig. 6. TEM images and particle size distribution histogram of AgNPs; Mole ratio of silver nitrate:m-hydroxybenzoic acid (A). 1:10 (B). 1:50; Scale bar $50 \mathrm{~nm}$

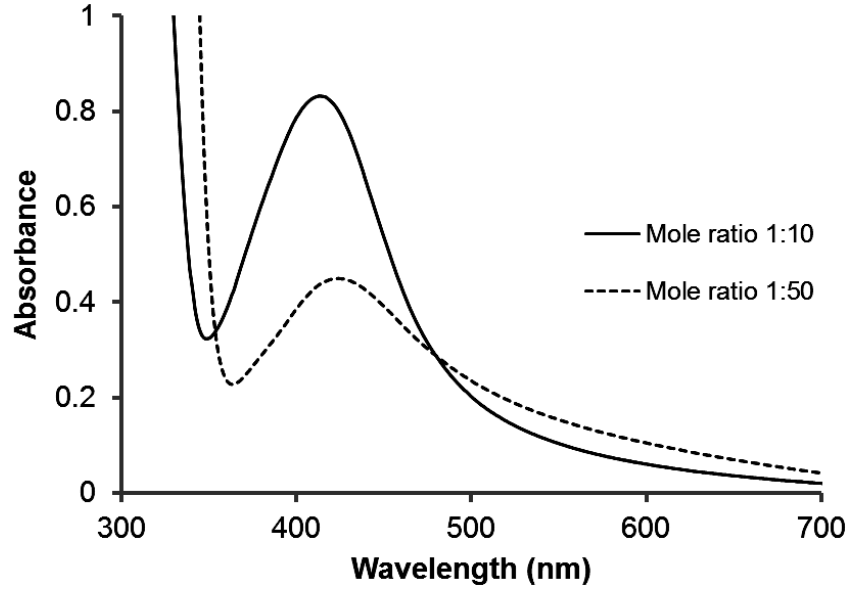

Fig. 7. Surface plasmon resonance spectra of AgNPs synthesized using different mole ratio of silver nitrate and $m$-hydroxybenzoic acid

\section{ACKNOWLEDGEMENTS}

This research was supported by BPPDN Scholarship by Directorate General of Higher Education, Ministry of Research, Technology and Higher Education, Republic of Indonesia.

\section{REFERENCES}

1. G. Lövestam, H. Rauscher, G. Roebben, B.S. Klüttgen, N. Gibson, J.P. Putaud and H. Stamm, Considerations on a Definition of Nanomaterial for Regulatory Purposes, Publications Office of the European Union, Luxembourg (2010).

2. G. Chen, I. Roy, C. Yang and P.N. Prasad, Chem. Rev., 116, 2826 (2016); https://doi.org/10.1021/acs.chemrev.5b00148.

3. M. Rycenga, C.M. Cobley, J. Zeng, W. Li, C.H. Moran, Q. Zhang, D. Qin and Y. Xia, Chem. Rev., 111, 3669 (2011); https://doi.org/10.1021/cr100275d.

4. C. Marambio-Jones and E.M.V. Hoek, J. Nanopart. Res., 12, 1531 (2010); https://doi.org/10.1007/s11051-010-9900-y.

5. S. Agnihotri, S. Mukherji and S. Mukherji, RSC Adv., 4, 3974 (2014); https://doi.org/10.1039/C3RA44507K.

6. B. Wiley, Y. Sun, J. Chen, H. Cang, Z.Y. Li, X. Li and Y. Xia, MRS Bull., 30, 356 (2005); https://doi.org/10.1557/mrs2005.98

7. P.C. Lee and D. Meisel, J. Phys. Chem., 86, 3391 (1982); https://doi.org/10.1021/j100214a025.

8. A. Panácek, L. Kvítek, R. Prucek, M. Kolár, R. Vecerová, N. Pizúrová, V.K. Sharma, T. Nevecná and R. Zboril, J. Phys. Chem. B, 110, 16248 (2006); https://doi.org/10.1021/jp063826h.

9. M. Annadhasan, T. Muthukumarasamyvel, V.R. Sankar Babu and N. Rajendiran, ACS Sustain. Chem. Eng., 2, 887 (2014); https://doi.org/10.1021/sc400500z.

10. L. Sulistiawaty, S. Sugiarti and N. Darmawan, Indo. J. Chem., 15, 1 (2015); https://doi.org/10.22146/ijc.21216. 
11. E. Susilowati, S.J. Triyono, S.J. Santosa and I. Kartini, Indo. J. Chem., 15, 29 (2015);

https://doi.org/10.22146/ijc.21220.

12. G. Gusrizal, S.J. Santosa, E.S. Kunarti and B. Rusdiarso, Int. J. ChemTech Res., 9, 472 (2016).

13. P.K. Jain, X. Huang, I.H. El-Sayed and M.A. El-Sayed, Plasmonic, 2, 107 (2007); https://doi.org/10.1007/s11468-007-9031-1.

14. A. Simic, D. Manojlovic, D. Segan and M. Todorovic, Molecules, 12 , 2327 (2007);

https://doi.org/10.3390/12102327.

15. M.C. Alvarez-Ros, S. Sánchez-Cortés and J.V. Garcia-Ramos, Spectrochim. Acta A Mol. Biomol. Spectrosc., 56, 2471 (2000); https://doi.org/10.1016/S1386-1425(00)00328-0.
16. S. Sánchez-Cortés and J.V. García-Ramos, J. Colloid Interface Sci., 231, 98 (2000): https://doi.org/10.1006/jcis.2000.7101.

17. P.J.G. Goulet and R.F. Aroca, Can. J. Chem., 82, 987 (2004); https://doi.org/10.1139/v04-075.

18. D.D. Evanoff Jr. and G. Chumanov, ChemPhysChem, 6, 1221 (2005); https://doi.org/10.1002/cphc.200500113.

19. Y. Sun and Y. Xia, Science, 298, 2176 (2002); https://doi.org/10.1126/science.1077229.

20. D.S. Sal'nikov, A.S. Pogorelova, S.V. Makarov and I.Y. Vashurina, Russ. J. Appl. Chem., 82, 545 (2009); https://doi.org/10.1134/S107042720904003X 